\title{
Ciudadanía y sustentabilidad ambiental en la ciudad: la recuperación del humedal Angachilla y la organización local en la Villa Claro de Luna, Valdivia, Chile
}

Juan C. Skewes. Universidad Austral de Chile, Valdivia, Chile.

Rodrigo Rebbein. Universidad Austral de Chile, Valdivia, Chile

Claudia Mancilla. Universidad Austral de Chile, Valdivia, Chile.

RESUMEN | La recuperación del humedal Angachilla por la junta de vecinos de la Villa Claro de Luna en la ciudad de Valdivia constituye un ejercicio donde se yuxtaponen la preocupación ambiental y el ejercicio de la soberanía ciudadana. Por medio de su organización, los residentes logran, por una parte, contribuir a la protección del medio ambiente; y por la otra, incrementar su participación en los procesos urbanos de una ciudad intermedia. A partir del redescubrimiento de las relaciones con la naturaleza, la comunidad amplía sus repertorios de significación y elabora nuevas formas de valorización del territorio basadas en la extensión de su cotidianidad y en las dinámicas de vida asociadas al paisaje natural, las cuales permiten la incorporación de la naturaleza a la ciudad.

PALABRAS CLAVE | ciudades intermedias, imaginarios urbanos, medioambiente urbano, participación ciudadana, transformaciones socioterritoriales.

\footnotetext{
ABSTRACT | Angachilla wetland recovery made by Villa Claro de Luna neighborhood association, in Valdivia, is an exercise that contributes to link environmental concerns with an exercise of citizen participation and local sovereignty. Through their organization, the community attains, on the one hand, environmental protection for a site that otherwise would remain as a wasteland; and, on the other, increases its participation in the urban processes of a midsize city. Since the rediscovery of the relation with nature, the repertoire of meanings and the elaboration of a new different kind of territory appreciation have grown, providing basis for the expansion of everyday life dynamics into a natural scenery, while allowing nature to become part of the city.

KEY WORDS | intermediate cities, urban imagery, urban environment, citizen participation, socio-territorial transformations.

Recibido el 31 de agosto de 2010, aprobado el 30 de mayo de 2011

Email: Juan C. Skewes, jskewes@uach.cl| Rodrigo Rehbein, rrehbeinm@gmail.com|Claudia Mancilla, cmancillam@gmail.com

Este artículo se inscribe en el marco del proyecto Fondecyt 1090465: "Los paisajes del agua: Prácticas sociales y sustentabilidad”. Agradecemos a quienes en distintas calidades han participado de este estudio. A la colega Claudia Monsalves y a los estudiantes de la práctica de etnografia urbana de la Escuela de Antropología de la Universidad Austral de Chile (segundo semestre de 2009): Fabián Coq, Andy González, Felipe Ortiz, Marcelo Piffaut y Arely Rojas. En forma especial, agradecemos a Jaime Rosales, presidente de la junta de vecinos Villa Claro de Luna durante el período en que se realizó este estudio. También a los evaluadores anónimos cuyos comentarios nos permitieron perfeccionar nuestra aproximación al tema.
} 


\section{Introducción}

La posibilidad de organizar la acción local a partir de una redefinición de la relación de la comunidad con la naturaleza en un medio urbano constituye una oportunidad para el desarrollo de la imaginación ambiental, la cual abre nuevos escenarios tanto de participación como de gestión local, a la vez que fortalece los vínculos orgánicos de la ciudad con su medioambiente. Así se desprende del análisis de la experiencia de la junta de vecinos de la Villa Claro de Luna, en Valdivia, en su lucha por la protección del humedal Angachilla.

A partir de la implantación del modelo neoliberal, la participación social urbana ha adquirido progresivamente un carácter subsidiario a la implementación de políticas sociales marcadas por un sesgo clientelista. En este contexto, la relación entre los actores locales y el Estado se hace instrumental, esto es, se sustituye la autoridad por la distribución de beneficios sociales (Peck, Leitner \& Sheppard, 2006). Desde el polo hegemónico, para asegurar la gobernabilidad se plantea dotar a la población de servicios esenciales, construir una forma de gobierno legítimo y potenciar el "pluralismo económico" (Zibechi, 2007). Importa, en consecuencia, (i) generar señales visibles de progreso que garanticen la credibilidad del sistema, (ii) asegurar una democracia formal para legitimar el modelo y, finalmente, (iii) promover una economía que por la vía del empleo asimile a sectores de la población que, en caso contrario, pudieran poner en jaque el orden social (véase, por ejemplo, Ferrero \& Gargantini, 2004; Lungo, 2005).

Este modelo adolece, entre otras debilidades, de una racionalidad instrumental que priva a los habitantes de un ejercicio real de voluntad política que se manifieste en la capacidad de organizar y gobernar los territorios que habitan (Alguacil, 2008). Los proyectos generados por los propios actores en función de intereses que desbordan lo meramente instrumental abren espacio a nuevas formas de participación que tienen el mérito de disputar a las autoridades urbanas parte de sus prerrogativas y ponerlas al servicio efectivo de la comunidad (Ferrero \& Gargantini, 2004). El caso de la Villa Claro de Luna representa una de tales iniciativas. Emplazada en los márgenes de una ciudad intermedia, los habitantes de esta comunidad se encuentran con la naturaleza mediante el humedal Angachilla que, desde la fundación de la villa en 2001 y hasta 2007, constituía un vertedero informal. Ese año, una nueva organización vecinal, especialmente motivada por un vecino interesado por la naturaleza, "descubrió" el humedal y comenzó a desarrollar iniciativas que propendían a su protección y recuperación. Semejante descubrimiento coincide con las nuevas formas de ciudadanía que actualmente se desarrollan en gran parte del mundo, y que recuperan la relación entre ciudad y naturaleza como su eje constitutivo (Gilbert \& Phillips, 2003).

La ciudad de Valdivia, gracias tanto a su exuberante entorno como a los conflictos surgidos en relación con el manejo de la naturaleza, provee un marco propicio para el surgimiento de una conciencia ambiental. La capital de la Región de los Ríos, situada a 840 kilómetros al sur de Santiago, se emplaza en la convergencia 
de los ríos Calle Calle, Valdivia, Cau Cau y Cruces, y su principal característica geográfica es la presencia de un número importante de cuerpos de agua y de humedales, tanto ribereños como interiores. La ciudad tiene una población de 127.750 habitantes, ubicándose como el decimoctavo centro urbano más poblado de Chile (Instituto Nacional de Estadística, 2005). Su explosivo crecimiento en las décadas de 1990 y 2000 ha estimulado la construcción de importantes conjuntos habitacionales, sobre todo hacia el sector sur poniente. Un ejemplo representativo de esta expansión son las villas con subsidio estatal de Los Alcaldes (construida entre 1992 y 2000), Los Ediles (construida entre 1999 y 2001) y Los Fundadores (construida entre 2000 y 2001), asentamientos que se encuentran próximos a importantes sistemas de humedales de la ciudad (Figura 1). Algunos de estos han sido rellenados para permitir tal expansión, lo que ha ocurrido con mayor frecuencia en los sectores urbanos de mayor plusvalía.

FIGURA 1 | Sector sur poniente de la ciudad de Valdivia

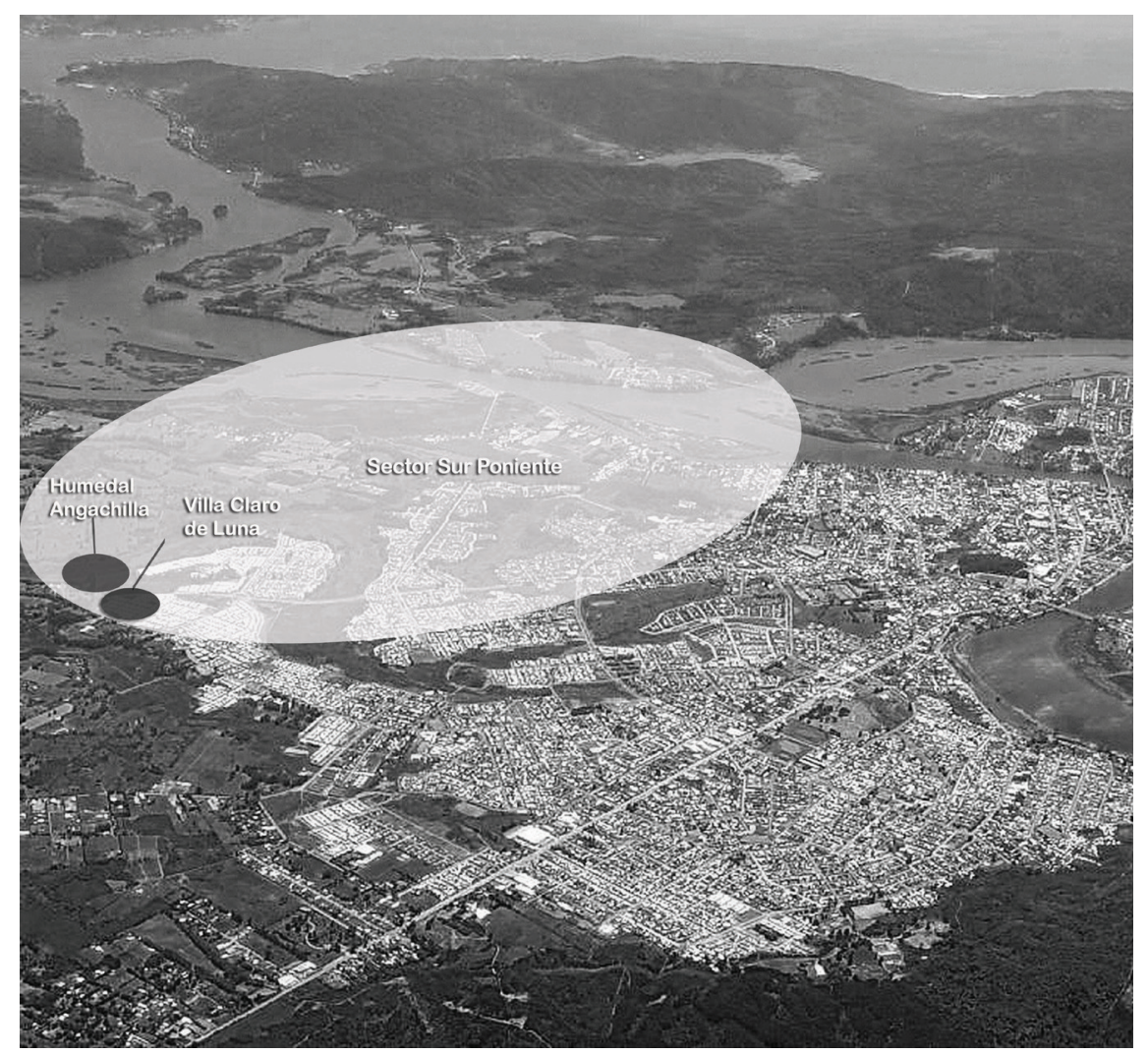

FUente Imagen intervenida a partir de fotografía aérea del Ministerio de Vivienda y Urbanismo, ReGIÓN DE Los Ríos, 2010. 
La experiencia de la Villa Claro de Luna se inscribe en el marco de una expansión urbana que, bajo la forma de intrusiones, se proyecta sobre humedales y cursos de agua. Este contexto deja en evidencia el entorno como parte estructurante del paisaje urbano y el esfuerzo de los residentes de la villa como una forma alternativa de constituir la relación ciudad-naturaleza y, de paso, asegurar la protección de sus derechos urbanos: frente a la voluntad de transformar en plusvalía el medio, la comunidad aspira a mejorar su calidad de vida reforzando su participación y, a la vez, protegiendo el medioambiente. Respecto de este último punto, la población de Valdivia mantiene un fuerte compromiso con su entorno, fortalecido por el hito del desastre ecológico del río Cruces (véase, por ejemplo, Cuenca, 2005). El Santuario de la Naturaleza "Carlos Anwandter" se convirtió en un referente de la defensa ambiental a nivel tanto nacional como internacional, cuando en el año 2004 se hizo pública la mortandad de cisnes de cuello negro (Cygnus melancoryphus) debido a la evacuación de residuos contaminantes en el río Cruces por parte de la Planta de Celulosa Valdivia, perteneciente a Celulosa Arauco y Constitución (CELCO S.A.). A partir de este caso, se resignificaron conceptos como "humedal" y "diversidad biológica", elementos que comenzaron a ser percibidos y asimilados como inherentes al entramado urbano valdiviano.

\section{Valdivia: ciudad de humedales}

Mientras la Región de los Ríos, con 136.678,7 hectáreas anegadas permanente o recurrentemente, es la cuarta región del país en cuanto a la presencia de humedales (Ministerio de Medio Ambiente, 2011; Osorio, 2009), del total del área urbana comunal, aproximadamente un 16\% (1.235,8 hectáreas) corresponde a zonas húmedas (Pulso Consultores, 2006), proporción que, en virtud de la expansión urbana de los últimos años, tiende a aumentar. En la actualidad se observa la expansión del manto urbano hacia su periferia, específicamente hacia los terrenos de la zona sur, sur poniente y sur oriente, que a su vez representan la superficie con mayor presencia del humedal valdiviano. A pesar de existir disponibilidad de zonas habitables en la ciudad, la alta demanda inmobiliaria ha llevado a una mayor presión por utilizar las zonas húmedas. En este contexto, el factor socioeconómico define el vínculo entre el precio del suelo y los proyectos que se realizan sobre él, siendo los terrenos del humedal valdiviano los más castigados y, desde una mentalidad de mercado, los más apetecidos para rellenados y el posterior desarrollo de proyectos inmobiliarios en ellos (Osorio, 2009).

Pese a las aparentes limitaciones que puedan imponer al desarrollo urbano, los humedales constituyen un importante recurso en términos medioambientales. A nivel mundial se los considera una riqueza ecológica amenazada: en España su superficie ha disminuido en un $60 \%$ en las últimas décadas, mientras que, en el mundo, han desaparecido en un 50\% desde 1900 a la actualidad (Corral, 2010). En el caso de Estados Unidos, se estima que a mediados del siglo XVI la superficie ocu- 
pada por humedales era de 87 millones de hectáreas, mientras que en la década de 1970 había disminuido a 40 millones, pérdida que en más del 50\% es consecuencia de las acciones humanas. En la actualidad, el Servicio de Fauna Silvestre (FWS) de ese país calcula que desaparecen 200.000 hectáreas de humedales cada año (Toledo, 2005). Seager (2000) sugiere que el desarrollo urbano ha eliminado más de la mitad de los humedales de Estados Unidos, a lo que cabe agregar la explotación maderera y la desecación con fines agrícolas como las mayores amenazas globales que enfrentan dichos sistemas.

Un humedal es definido como un ambiente de tierras bajas cuya superficie se encuentra anegada permanente o intermitentemente, situación que da origen a suelos anóxicos ricos en carbono orgánico, con el correspondiente desarrollo y mantenimiento de fauna endémica y diferenciada de las zonas adyacentes (Díaz et al., 2006). Como ecosistema, depende de una constante o recurrente inundación superficial o saturación a nivel o cerca de la superficie del sustrato, lo que constituye su característica principal, junto con rasgos biológicos, químicos o físicos suscitados por el anegamiento. Las características diagnósticas de un humedal son los suelos hídricos y la vegetación hidrofítica, elementos que están presentes a no ser de su remoción por factores fisioquímicos, bióticos o antropogénicos (National Research Council, 2001). Un acuerdo internacional para proteger estos sistemas es la Convención Ramsar sobre los Humedales, cuyo objetivo principal es proteger, por su riqueza biológica, estas extensiones de marismas, pantanos y turberas, o superficies cubiertas de aguas, de régimen natural o artificial, permanentes o temporales, estancadas o corrientes, dulces, salobres o saladas, incluidas las extensiones de agua marina cuya profundidad en marea baja no exceda de seis metros (Ramsar, 1994).

Los humedales proveen servicios ecosistémicos fundamentales para la biodiversidad: por su capacidad para la retención de sedimentos y nutrientes constituyen uno de los ecosistemas más productivos, sirviendo de soporte a una gran cantidad de especies. Aproximadamente el $40 \%$ de las especies del mundo habita humedales, en tanto que un $12 \%$ de las especies animales lo hacen en humedales de agua dulce. Dos tercios de las especies marinas de consumo humano dependen de los humedales en alguna etapa de su ciclo biológico. Otros recursos naturales que se encuentran en estos ambientes son los moluscos y crustáceos, peces, plantas medicinales, forrajes y frutas. Los humedales permiten, también, controlar las inundaciones, al acumular las aguas que, de lo contrario, anegarían las zonas donde se ubican. Por otra parte, alimentan los acuíferos subterráneos, donde se conserva cerca del 97\% de las aguas dulces no congeladas del mundo. Los humedales ayudan a mitigar los efectos del cambio climático al almacenar gases del efecto invernadero fijados en la materia orgánica. Por otra parte, poseen una importancia y significación que trasciende la materialidad del paisaje, sustentada en aspectos culturales de orden histórico, religioso y arqueológico que en muchos casos los definen como lugares patrimoniales y de gran atractivo turístico (Rojas, Campos, Alpízar, Bravo \& Córdoba, 2003; Tiner, 2005). 
Los humedales constituyen uno de los elementos más característicos de la morfología urbana de la ciudad de Valdivia, al abarcar grandes extensiones de territorio. En ella es posible distinguir tres categorías de zonas húmedas que conforman humedales en tanto sistema ecológico metapoblacional interconectado con características de corredor de especies, hualves o áreas deprimidas cerradas cuyos límites son tanto naturales como antrópicos, y vegas o planicies adyacentes a los cauces que se inundan según los regímenes de marea. De acuerdo con este criterio, en la ciudad los hualves alcanzan 21 hectáreas; los humedales, 185 hectáreas y las vegas, 260 hectáreas, con una superficie total de 466 hectáreas (Pulso Consultores, 2006), que corresponde a un 23,01\% del área total urbana de 2.025 hectáreas (Osorio, 2009). En la ciudad existen alrededor de nueve humedales constituidos y delimitados: Angachilla, El Bosque-Angachilla, Errázuriz, Isla Teja, Islote Haverbeck, Kramer, Las Ánimas, Las Marías-Río Cau Cau, Miraflores y Río Cruces (Pulso Consultores, 2006).

FIGURA 2 | Distribución de zonas húmedas en la ciudad de Valdivia

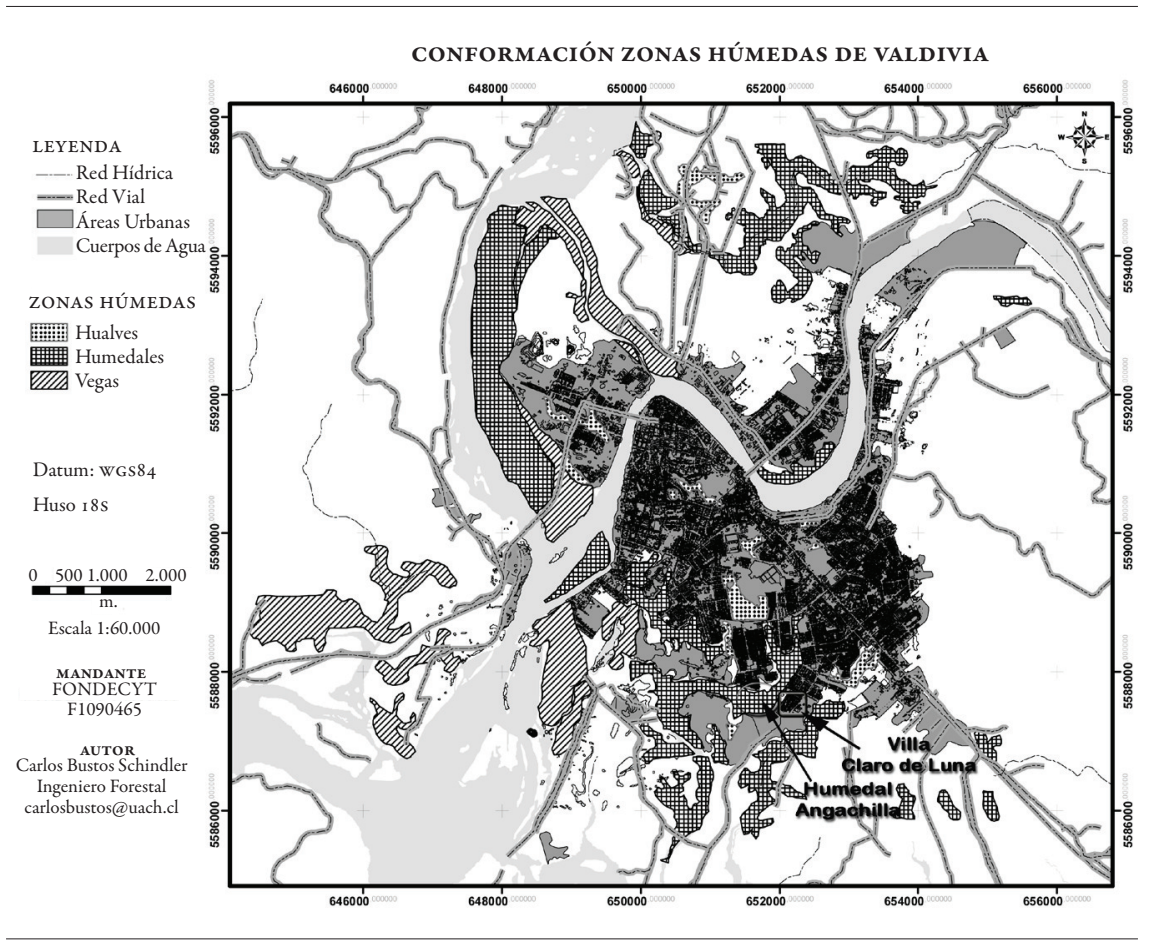

fuente Proyecto Fondecyt F1090465, Los Paisajes del Agua.

Los humedales ribereños localizados en Valdivia presentan en su plano una cubierta vegetal baja, con predominio de pasto y matorral arbustivo y presencia de arborización en sus bordes aguas arriba. En los humedales interiores se aprecian pastos y matorral arbustivo con una importante vegetación arbórea en sus bordes, 
cuyo entorno corresponde a zonas de viviendas y vialidad que limitan directamente con el humedal (Pulso Consultores, 2006).

Los ríos, humedales y, en general, los espacios húmedos de Valdivia moldean el tejido urbano y demarcan los espacios, condicionando el diseño y la planificación a las formas de la naturaleza. ${ }^{1}$ Sobre estos espacios se construyen diversos significados asociados a los estilos de vida de la ciudad y a su apropiación para uso habitacional, influidos por las características de la habitación propias de cada segmento socioeconómico. Los humedales contiguos a sectores de bajos ingresos han sido usados como vertederos clandestinos, escondrijos y habitación eventual. En cambio, aquellos ubicados en sectores de mayor plusvalía urbana han sido rellenados para la construcción de viviendas y, en los últimos decenios, también han sido aprovechados para la habilitación de parques, áreas verdes y de contemplación escénica.

\section{El movimiento ciudadano en el rescate del humedal Angachilla}

El ejercicio de la ciudadanía urbana en torno a las reivindicaciones ambientales no es patrimonio exclusivo de la Villa Claro de Luna. Así lo demuestran los movimientos generados en torno a la provisión de servicios, al acceso y calidad del agua y a la protección de los recursos ambientales en los medios urbanos (véase, por ejemplo, Gervais-Lambony, Landy \& Oldfield, 2005; Gilbert \& Phillips, 2003; von Bertrab \& Zambrano, 2010).

El movimiento para la defensa del humedal de Angachilla, en el caso chileno, fue motivado por la denuncia relativa al vertedero clandestino. Este hecho marcó una transición importante en las inquietudes de la población en relación con su entorno, giro que llevó a que el espacio urbano y su emplazamiento en la geografía local adquirieran un nuevo significado y la acción colectiva se reorientara hacia nuevos fines. De hecho, hasta el año 2005 la preocupación principal de las y los vecinos había estado centrada en la delincuencia, el alcohol y las drogas, temas de referencia permanente en los medios de comunicación local.

La Villa Claro de Luna se ubica a doscientos metros del humedal Angachilla; corresponde a una población nacida del Programa Especial de Viviendas para Trabajadores, PET (D.S. No 235/1985), constituida por residentes provenientes de los sectores urbanos populares tradicionales de Valdivia, principalmente de la extensa población Corvi, de los años 60. Claro de Luna consta de tres edificios con doce departamentos cada uno y 205 viviendas familiares, con un total estimado de ochocientas personas. Su población está vinculada principalmente al trabajo en el comercio y los servicios tanto formales como informales. Sus habitantes, mediante la junta de vecinos $\mathrm{N}^{\circ} 75$, se han constituido en una fuerte organización social que constantemente ha buscado mecanismos de recuperación y preservación del humedal, con el fin de implementar un espacio de encuentro e identificación entre sus pobladores.

El emplazamiento de la población, en un entorno que con los años incrementaría el número de viviendas sociales, provocó el temor entre los vecinos por la delincuencia,

1 Guarda (1980) singulariza Valdivia señalando: "El emplazamiento mismo, en un meandro formado por la confluencia de los claros cauces del Calle Calle y el Valdivia (...) hacían de su estampa un escenario único” (p. 16). 
preocupación que terminó por inhibir la acción colectiva en el primer quinquenio de vida de la junta vecinal. El activismo ambiental de la Villa Claro de Luna adquirió fuerza el año 2007, cuando los vecinos se unieron para detener el vertedero clandestino formado desde hacía algunos años en el humedal. ${ }^{2} \mathrm{Al}$ fomento de la conciencia ambiental contribuyó el creciente movimiento ciudadano que, como se ha señalado, alcanzó su máxima expresión en Valdivia por la defensa del Santuario de la Naturaleza "Carlos Anwandter".

La presencia de gran cantidad de escombros y residuos en el humedal no solo generó entre los vecinos una creciente percepción de riesgo en términos de salud pública, sino que los expuso a una situación de vulnerabilidad ligada al consumo de alcohol, drogas y delincuencia presentes en ese espacio. Desde la perspectiva de los vecinos, el deterioro ambiental proyectaba una imagen negativa hacia el resto de la ciudad, asociada a la marginalidad y abandono, y con ello se socavaba la identidad colectiva y el sentido de pertenencia, proceso que, a su vez, era consistente con la ubicación periférica de la villa (Ortiz et al., 2009). El medio y los significados implicados en él afectaban la percepción de la calidad ambiental y la buena vida (Rapoport, 1977).

La lucha contra el vertedero constituye un hito a partir del cual se han realizado diversas actividades en defensa del lugar, entre las que destacan jornadas de limpieza, plantación de árboles nativos, talleres de educación ambiental y reciclaje para niños y adultos, conciertos y la reciente construcción de un vivero intercultural. ${ }^{3}$ Mediante su lucha, la comunidad local ha redescubierto el espacio con el que había convivido y que hasta ese momento le era ajeno y amenazante. Este encuentro la ha llevado a desarrollar diversas acciones por intermedio de las que se manifiesta el sistema de valores puesto en juego a partir de la nueva relación con el entorno. Su forma de actuar traduce su nueva forma de percibir y de conocer (Vargas \& Piñeiro, 2005). A partir del "descubrimiento" del humedal por parte de los vecinos, se ha reconfigurado la acción colectiva en términos de su organización y de sus bases ideológicas. La reivindicación local pasó de ser una demanda por la protección policial, a otra que planteaba cogestionar la protección del humedal y en torno a la cual se generó una red de iniciativas que aguardaron este momento para su expresión. En este sentido, los paradigmas de la gestión se vinculan a una cosmovisión, a un sistema de ideas sobre el habitar urbano, las que se gestan en el seno de la cultura.

Una de las principales fortalezas del movimiento, reconocida por los propios sujetos, se refiere a la capacidad de organización y gestión participativa, aspecto fundamental en el desarrollo de la ciudadanía de la Villa Claro de Luna. Mediante tal capacidad ampliada, han desarrollado un proceso de intercambios con el humedal que ha permitido ampliar su sistema de referencias para la percepción e inte-

2 El sábado 24 de mayo de 2007 se organizó una asamblea extraordinaria en la plazuela de la Villa Claro de Luna en la que participaron como invitados el diputado Alfonso de Urresti, el secretario regional ministerial del Ministerio de Vivienda y Urbanismo, Alejandro Larsen; y el director regional del Servicio de Vivienda y Urbanismo, Germán Sáez. Entre las propuestas de la junta de vecinos se destacan: mayor participación ciudadana, una sede vecinal, mejorar la calidad de vida, recuperar los espacios públicos e incrementar la inversión social en el sector (Blog Villa Claro de Luna, 28 de mayo de 2007)

3 Algunas de estas actividades están documentadas en el blog de la organización local, http://villaclarodeluna.blogspot.com 
racción con ese entorno. La participación es, en este sentido, un proceso en que la comunidad se compromete con la transformación de su propia realidad asumiendo las tareas que le corresponden, de manera que el movimiento deja de ser una suma de aventuras individuales para expresarse colectivamente tanto mediante proyectos solidarios y propuestas comunes, como del aprendizaje de nuevas relaciones sociales y modos de vida y de la instauración de una nueva visión de las cosas (Corvalán $\&$ Edy, 2003). Estas propuestas, surgidas de la propia comunidad, representan respuestas a una demanda socialmente reconocida, la cual contribuye a la constitución de una identidad colectiva que favorece el surgimiento de nuevos esquemas culturales, los mismos que a su vez sustentan tales formas de actuar.

El constante protagonismo de los vecinos en la recuperación del humedal como reserva natural urbana ha llevado a un reconocimiento de esta experiencia en otros puntos de Valdivia y de Chile; no obstante, el proyecto de cogestión no se ha materializado, al no concretar la autoridad pública la cesión en comodato del área del humedal, tal cual fuera comprometido por el Servicio de Vivienda y Urbanismo (SERVIU) el 29 de mayo de 2009 (Gobernación de Valdivia, 2009). Así, en octubre de 2008 los dirigentes de la junta de vecinos de la Villa Claro de Luna organizaron el primer coloquio ciudadano sobre patrimonio ambiental en Valdivia, denominado "Desde los humedales: una mirada participativa para construir ciudad", instancia apoyada por el Centro Educacional San Nicolás, la Dirección de Extensión y la Escuela de Artes Visuales de la Universidad Austral de Chile (UACh), la Agrupación de Mujeres Manos Divinas, el jardín infantil Caracolito, la Escuela de Lenguaje San Miguel, la Escuela Angachilla, la agrupación de vecinos Angachilla y la Corporación Servicio Paz y Justicia (SERPAJ). El año 2009, los dirigentes de la junta de vecinos participaron en los seminarios "Diálogos sobre política, pobreza y exclusión social", y "Parques urbanos, una necesidad del siglo XXI", de la Universidad de Chile y Universidad Central, respectivamente. En ambas iniciativas expusieron sobre su trabajo comunitario y la creación de reservas naturales urbanas en Valdivia. En junio de ese mismo año, durante la conmemoración del mes del medioambiente, la Comisión Nacional del Medio Ambiente (CONAMA) de la Región de los Ríos entregó un reconocimiento a la junta de vecinos de la Villa Claro de Luna por su gestión ambiental.

Entre los diversos proyectos ejecutados por la organización vecinal de la Villa Claro de Luna asociados a la recuperación del humedal Angachilla, se encuentra "Parque Humedal", en el año 2008, financiado por el Programa de Desarrollo Social Autogestionado, del Fondo Solidario e Inversión Social (FOSIS). Durante 2010, la junta de vecinos desarrolló el proyecto "Restauración y conservación de la biodiversidad del humedal Angachilla mediante la creación de una Reserva Natural Urbana", financiado por el Fondo de Protección Ambiental de CONAMA, iniciativa que busca habilitar el humedal como patrimonio ecológico de la ciudad de Valdivia y mejorar la calidad de vida de sus habitantes. Las actividades contempladas en este proyecto incluyen despeje, recuperación y señalética de senderos; taller de nivel básico en reconocimiento, identificación y clasificación de plantas nativas; jornada de restauración con especies nativas; cercado perimetral del humedal y señalética de la Reserva Natural Urbana; talleres de identidad comunitaria, entre otras. 
En la actualidad, la comunidad sigue gestionando la conservación del humedal y consolidando un discurso sobre este espacio como un lugar para la conservación de la biodiversidad y de recreación para la comunidad valdiviana. Así, el día 24 de mayo de 2011 los vecinos de la villa constituyeron el Comité Ecológico Angachilla, cuyos objetivos principales son impulsar acciones conducentes a la preservación y cuidado del humedal Angachilla como Reserva Natural Urbana de la ciudad de Valdivia, junto con promover valores humanistas resultantes de la práctica y desarrollo de actividades educativas y formativas que tiendan a promover y proteger el respeto a las personas por la biodiversidad y el cuidado del medioambiente (Diario Cultural, 26 de mayo de 2011). El conjunto de estas acciones fortalece el sentido de identidad y pertenencia al sector, además de la articulación de las diversas organizaciones locales. ${ }^{4}$ La reivindicación por el humedal genera dinámicas y relaciones de poder en el territorio y se asocia a la reorientación cognitiva que supone agruparse en torno a la belleza escénica del medio en que se habita. Como consecuencia de ello, se crean modalidades icónicas alternativas para representar el bienestar residencial. En el siguiente esquema se pone de relieve el conglomerado de relaciones desencadenadas luego de tres años de organización popular:

La dinámica local se desencadena a partir de un cambio de percepción que va desde la inseguridad ciudadana hacia la oportunidad del humedal. El cambio se traduce en un conjunto de nuevos valores y conceptos (como interculturalidad o reserva urbana), a partir de los que la organización se redefine hacia su interior estableciendo nuevas alianzas para disputar el espacio donde se adoptan las medidas de intervención en el espacio urbano. La defensa del humedal pone, así, en tela de juicio el concepto dominante de "área verde" que promueve la autoridad urbana, al tiempo que desencadena tensiones cuya raíz se relaciona con la soberanía local de los residentes frente a imposiciones externas. El modelo de área verde propuesto tanto por las inmobiliarias como por el propio Estado involucra habilitación de espacios e inversiones en infraestructura contrarias a la armonización de los espacios humanos y no humanos (Weingartner, 2001). La propuesta de la organización local apunta a la noción de reserva natural urbana. ${ }^{5}$

Las concepciones levantadas por la organización contradicen los intereses dominantes en el planeamiento de la autoridad urbana, de modo que la defensa del humedal, al tiempo que promovía ideas alternativas para el uso del espacio público, no tardó en encontrar obstáculos. En abril de 2010, la entrada del humedal fue incendiada por desconocidos, mientras que en mayo del mismo año, maquinaria pesada intervino el humedal para extraer tierra y depositar los desechos de las obras de pavimentación de la avenida Pedro Montt, acción que ocasionó grandes impactos en la biodiversidad de Angachilla.

4 El fortalecimiento de la identidad local mediante la acción organizada ha sido descrito por Gissi y Soto (2010).

5 "Las Reservas Naturales Urbanas son fragmentos de ecosistemas naturales que quedan al interior de las ciudades en el proceso de expansión y desarrollo urbano. Estas áreas nos proveen de servicios ambientales clave para el urbanismo sostenible, como el drenaje y depuración de aguas de lluvia, la depuración del aire, la mitigación de contaminación acústica y lumínica, o la regulación térmica, entre otras. Unido a ello, estas áreas facilitan el acceso y disfrute de la naturaleza a todos los ciudadanos, y en especial para los grupos más vulnerables” (Blog Villa Claro de Luna, 2009). 


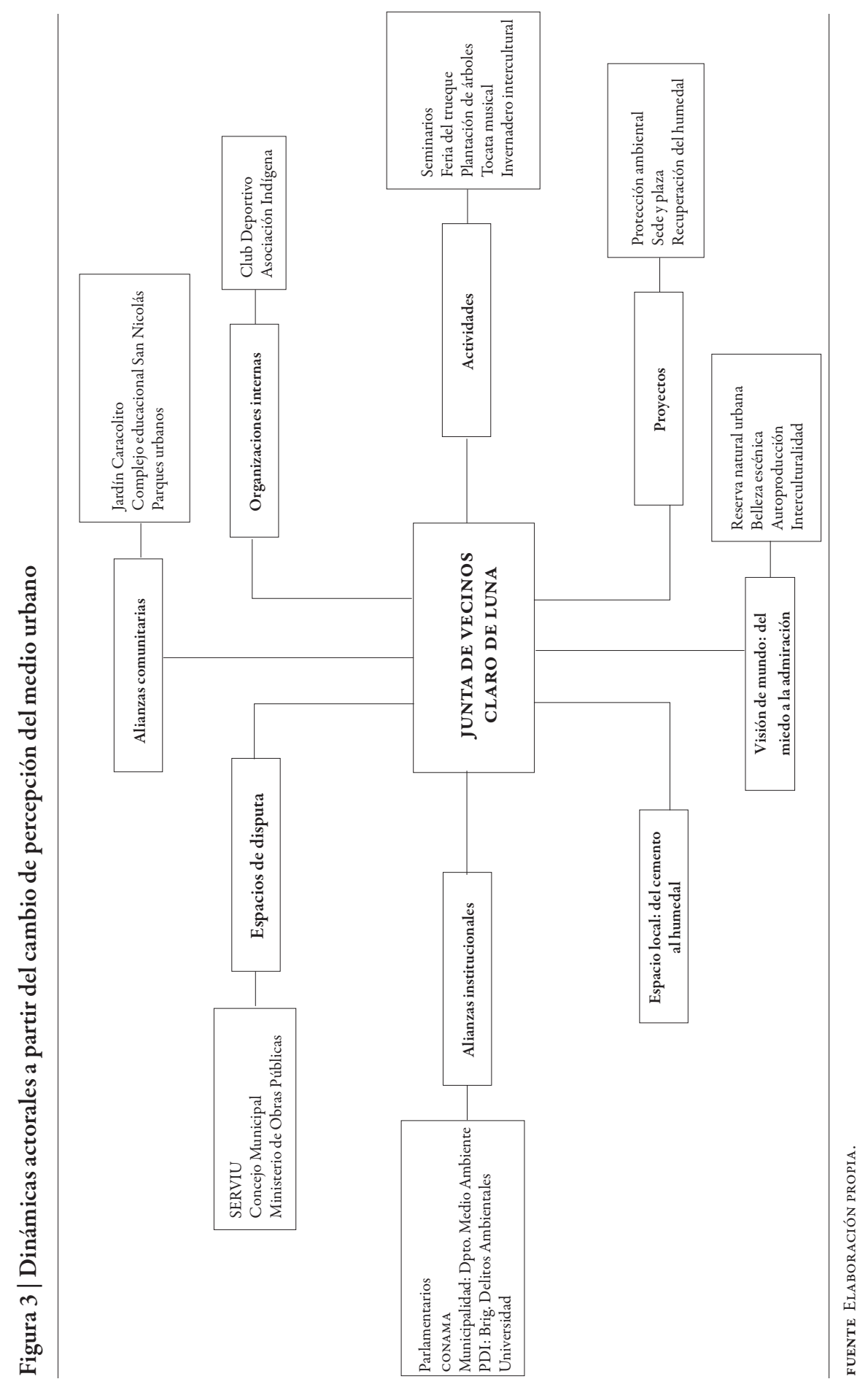


La alta demanda inmobiliaria y la creciente tendencia de expansión de la ciudad hacia la periferia han llevado a que los vecinos de la Villa Claro de Luna vean amenazada su organización a partir de la disputa de dicho espacio con las diversas autoridades encargadas de la planificación territorial, a saber, el Servicio de Vivienda y Urbanismo (SERVIU), el Ministerio de Obras Públicas (MOP) y el Concejo Municipal de Valdivia. Según los vecinos, el principal punto de conflicto se vincula al nuevo plano regulador, que, pese a tipificar Angachilla como zona de humedal - es decir, como un área de protección ambiental-, proyecta la extensión de la avenida Circunvalación por sobre ella. Dicho proyecto busca descongestionar el núcleo urbano central, agilizando el flujo vehicular de los que circulan de norte a sur por el anillo externo de la ciudad. La construcción y habilitación de la avenida Circunvalación exige el relleno de gran parte del humedal, opción que afecta la motivación inicial de la comunidad respecto de su entorno y calidad de vida. Muchas de las áreas húmedas de la ciudad, como los hualves, representan la continuación de los humedales que han sido intervenidos por la acción antrópica, especialmente por la Dirección de Vialidad (PULSO, 2006, p. 18). Esta situación ha demarcado las relaciones de conflicto entre la comunidad local y las autoridades responsables de la materia, ya que estas últimas anteponen criterios administrativos y de planificación urbana, a los intereses y niveles de valoración que la comunidad ha construido sobre estos espacios.

De forma paralela a dichos conflictos, se han establecido alianzas con diversas instituciones públicas y otros organismos relacionados con el medioambiente, como la Comisión Nacional del Medio Ambiente (CONAMA), la Corporación Nacional Forestal (CONAF), la Municipalidad de Valdivia y su Departamento de Medio Ambiente y Conservación; también con algunos parlamentarios, con la Brigada de Delitos Ambientales de la Policía de Investigaciones (PDI) y con la Universidad Austral de Chile, que colaboran permanentemente en las diversas actividades. Además, las actividades son abiertas a la comunidad valdiviana y difundidas por los medios de comunicación local.

A partir de esta difusión, los vecinos de la Villa Claro de Luna han buscado generar la identificación de los sujetos con el espacio para fortalecer el desarrollo de políticas de autogestión. Así, uno de los impactos más relevantes de la acción social sobre otros actores de la ciudad ha sido la introducción de la noción de "reserva natural urbana" en el imaginario tradicional de ciudad. De esta manera se busca que el humedal Angachilla y otras zonas húmedas de Valdivia sean reconocidos bajo esa categoría, y se propone que la protección de estos espacios y de los bosques del interior de la ciudad no queden subordinados a los criterios de urbanización planteados por la autoridad, ya que en su conjunto pueden generar una mirada de ciudad sostenible y convertirse en un eje de atracción turística.

\section{Sobre el imaginar y habitar el espacio}

La recuperación del humedal Angachilla ha llevado a complejizar los procesos de interacción con el paisaje, fenómeno a partir del cual surgen nuevas visiones y oportunidades de mejoramiento en la calidad de vida y desarrollo, las mismas que se ma- 
nifiestan por el discurso ambiental de la comunidad. El "descubrimiento" del humedal representa una transición radical en la imaginación urbana de quienes gestan la organización a partir de una reivindicación de carácter paisajístico, que gravita sobre la definición identitaria, la red de articulaciones y el universo de acción por el que se responde a las nuevas demandas. Este imaginario ambiental o paisajístico ${ }^{6}$ se sustenta en la capacidad de los sujetos de tomar conciencia e interactuar con la realidad material del territorio en el que habitan. Tal imaginación material permite que los residentes participen en la construcción de los nuevos paisajes, proceso al que concurre la totalidad de las formas del conocer humano - científicas, estéticas, éticas, religiosas, míticas - y que sintetiza en la acción local la multidimensionalidad natural y cultural del paisaje (véase, por ejemplo, Gervais-Lambony, Landy \& Oldfield, 2005; Toledo, 2006).

La acción local representa una reorganización del sistema codificante que, mediante procesos de selección, incorpora nuevos contenidos y códigos culturales (Lotman \& Uspenskij, 2000). El conflicto que moviliza a los vecinos de Claro de Luna muta el repertorio de significaciones y sistemas de relación en torno al humedal como espacio de la naturaleza, jerarquizando y reactualizando los conocimientos y usos que permanecían dormidos en la memoria colectiva o que son simplemente incorporados y creados por la colectividad. Este proceso lleva a una toma de conciencia estética del territorio y a la internalización de nuevos conocimientos que complejizan la percepción del paisaje (Aasbø, 1999). Como plantea Le Breton (2007), experimentar el mundo no es estar con él en una relación errónea o justa; es percibirlo con su estilo propio, en el seno de una experiencia cultural, ya que las cosas nunca pueden ser separadas de los sujetos que las perciben, humanizándolas y trascendiendo su materialidad, y con ello transformando los territorios en un espacio culturizado y de constatación simbólica de la realidad.

La internalización de estos nuevos repertorios culturales permite consolidar el discurso ambiental propio de los vecinos, materializado en las distintas acciones y estrategias participativas emprendidas para la protección del humedal, una puesta en valor que engloba las expectativas y los niveles de percepción en un proceso continuo de intercambio con el humedal. Este discurso integra y homogeneiza valores culturales, sociales, económicos, estéticos y afectivos que han sido expresados en la praxis del movimiento y que, desde la perspectiva de los actores, han permitido superar la marginalidad proyectada hacia el resto de la comunidad valdiviana.

El dinamismo con que se generan estas significaciones se vincula con el dinamismo de la vida social y cultural, ya que - como señalan Lotmann y Uspenskij (2000) - para el ser humano la movilidad del medio y sus condiciones cambiantes constituyen un estado normal de la existencia, un cambio en el modo de vida. La acción colectiva lleva a una definición de necesidades y expectativas que, en este caso particular, se sustentan sobre la premisa de que alcanzar una mejora en la calidad y estética del paisaje lleva a un avance en la calidad de vida.

Los distintos niveles de valorización asociados al paisaje, condensados en el discurso ambiental de la comunidad, se inscriben y movilizan en el campo de la interacción de los sujetos con su territorio. Para Baeza (2003), la relación con la

El término "imaginario" se utiliza de acuerdo con la propuesta de Cornelius Castoriadis (1987). 
realidad, el entorno y las demás personas, no es una cuestión exclusivamente sensorial, ya que no es posible interpretar aquello que es externo sin tener que significarlo en el acto mismo de la toma de contacto con ese algo, realizada por el sujeto que experimenta su externalidad. Por consiguiente, la significancia no es otra cosa que la mediación necesaria que se interpone entre la comunidad y lo que la rodea. Esta significancia práctica es el decodificador que, con fines prácticos, se debe ejecutar para operacionalizar informaciones sin accesibilidad comprensiva inmediata. Así, la asignación de significados es la condición misma de reducción de la complejidad intrínseca de las cosas. La intercalación de una percepción estética del medio lleva a una reelaboración de los entendimientos prevalentes en el entorno barrial y a la posibilidad de formas nuevas de inteligibilidad del mundo urbano.

El análisis del caso aquí presentado sugiere integrar la dimensión del paisaje imaginado como dimensión analítica para comprender los procesos de valorización de los territorios. Esta imaginación se imbrica con las dimensiones física y perceptiva de los paisajes, las mismas sobre las cuales la comunidad proyecta sus expectativas, necesidades y visiones del territorio, elaborando códigos de significación mediante los cuales se integran la realidad social y cultural con los espacios a un nivel de significancia práctica. El paisaje imaginado se convierte en un prisma, un elemento de mediación que moldea la forma en que la acción local se relaciona con la materialidad del paisaje y con los modelos culturales prevalentes de percepción de dicho espacio.

Las etapas del proceso de la acción local que se desprenden de este análisis se pueden enumerar del modo siguiente:

1) El punto de referencia. En esta etapa se introduce un elemento de cambio en la normalidad de la interacción cotidiana entre los sujetos y el espacio, a partir del cual los sujetos cambian su perspectiva de interacción. Por ejemplo, la visualización y percepción de consecuencias de un desastre ambiental.

2) Actualización de los códigos culturales de significación y adquisición de nuevos conocimientos en torno a la naturaleza.

3) Surgimiento de un imaginario ambiental. En él se condensan las distintas visiones y niveles de valorización del paisaje que coexisten en un grupo determinado. A partir de dicho imaginario se elabora el discurso ambiental de la comunidad.

4) Consolidación del movimiento ciudadano. El discurso ambiental se expresa en la praxis del movimiento, y mediante este movimiento el paisaje es reintegrado a la vida cotidiana y ciclos de vida de los sujetos, portando nuevas formas de valorización.

En este proceso, el humedal de Angachilla no solo se presenta como un elemento configurador de las formas urbanas, sino también como referencia para la construcción de sentido y, con ello, como soporte para la organización de una acción orgánica respecto del contexto territorial, garantizando así la característica imbricación de ciudad y naturaleza del paisaje valdiviano. 
Esta concepción de habitar que aquí se ha desplegado, basada en una integración del entorno natural y las formas urbanas, transforma el espacio en una extensión de la cotidianidad y de la dinámica de vida de vecinos y vecinas, y mediante ella se redescubre la relación con la naturaleza. Aprender a percibir e interactuar con el humedal ha implicado integrarlo como elemento constitutivo de la comunidad, lo ha transformado en un tejido familiar y coherente sobre el que se articula el complejo entramado de referencias sociales y culturales.

La experiencia de la Villa Claro de Luna no es excepcional: en diversas partes del mundo hay iniciativas cuyos objetivos son similares. En España, por ejemplo, el Plan Andaluz de Humedales busca garantizar la conservación y la restauración de los procesos ecológicos clave que determinan la integridad de los humedales de Andalucía por intermedio de la participación ciudadana, para lo cual experimenta con nuevos modelos de gestión cuya finalidad es la convivencia armónica y equilibrada entre la comunidad y su medio (Molina, Montes, González, Rubio \& Comisión de Seguimiento de la Gestión de los Humedales, 2002). El cambio en la apreciación del territorio abre, pues, la posibilidad de incorporar a la comunidad local en la gestión del paisaje. Para De San Eugenio (2006), la delimitación de los valores simbólicos e identitarios del paisaje por parte de los "no expertos" resulta fundamental en el diseño de líneas de acción respetuosas con el medioambiente y la singularidad de los paisajes. La participación pública se concebiría por intermedio de la integración de las opiniones ciudadanas a los procesos de evaluación y generación de conocimiento sobre los espacios que habitan. Mediante esta etapa de traspaso de poder desde los modelos expertos hacia los no expertos es posible integrar las necesidades e intereses de las personas, haciendo emerger el valor social y cultural de los paisajes por sobre los criterios de la arquitectura y urbanismo, y se puede movilizar a las comunidades locales.

El movimiento social generado en la Villa Claro de Luna pone de manifiesto la necesidad de incorporar la participación ciudadana en el desarrollo y gestión de políticas para la planificación de los espacios urbanos, asegurando así los estándares ambientales y la calidad de vida. Dicha integración debe ser vista como un proceso continuo que facilita tanto los ajustes de la gestión en relación con las dinámicas de la vida social, como la jerarquización continua de los tipos de valoración del paisaje que los sujetos expresan mediante los discursos.

\section{Conclusiones}

La articulación de la demanda urbana a la dimensión paisajística supone una transformación radical del programa de acción, de la orientación general y de la relación entre residentes y su entorno, tal cual se desprende del caso de la junta de vecinos Villa Claro de Luna. Este proceso desencadena nuevos contenidos y códigos culturales aplicables a la comprensión de las relaciones que ocurren tanto al interior de la comunidad, como entre esta y los otros actores urbanos y el medio institucional. Este ejercicio se inscribe en el marco de nuevas territorialidades donde se reconstruyen 
los movimientos populares urbanos de modo autónomo, donde la organización de la vida cotidiana se asume sobre las bases de la colaboración y una relación horizontal con la naturaleza. Esta relación de horizontalidad está mediada por un conjunto de procesos de participación e interacción con el espacio, a partir de los cuales se construyen y circulan las nuevas formas de valorización del territorio que generan beneficios recíprocos entre la comunidad y la naturaleza. En el caso de Claro de Luna, a partir de esta relación se inauguró una fase de restauración y conservación del hábitat de especies endémicas y vegetación nativa, proceso que amplió el espacio de socialización e interacción entre los habitantes de la villa, transformando el humedal en el eje de articulación de un proyecto común para mejorar las condiciones de vida y enfrentar la exclusión y marginalidad de la periferia de la ciudad.

El imaginario ambiental, surgido a partir de la ampliación de los sistemas de referencias y códigos culturales de significación, permite insertarse en la gestión territorial mediante un movimiento que lleva a desestructurar y reestructurar la institucionalidad, actuando como un elemento configurador del espacio a partir del paisaje imaginado. Estas nuevas formas de imaginación son consistentes, por una parte, con un ejercicio colectivo que desplaza las fronteras de los derechos urbanos y ambientales como convencionalmente son definidos, hacia el ejercicio de una ciudadanía socioecológica (Gilbert \& Phillips, 2003); y por otra, abre las puertas a la tarea práctica de conservar y restaurar ecosistemas amenazados (von Bertrab \& Zambrano, 2010). En Valdivia ello ha abierto la posibilidad de gestionar de modo alternativo el territorio y responder a desafíos que no han sido considerados por la planificación urbana ni por los organismos públicos, lo que a su vez ha reforzado un proyecto de integración orgánica de la ciudad y su paisaje. ${ }^{7}$ IEURE

\section{Referencias}

Aasbø, S. (1999). History and ecology in everyday landscape. Norsk Geografisk Tidsskrift - Norwegian Journal of Geography, 53, 145-152. doi: 10.1080/00291959950136867

Acselrad, H. (1999). Sustentabilidad y ciudad. EURE, 25(74), 35-46. doi: 10.4067/S0250

$-71611999007400003$

Alguacil, J. (2008). Espacio público y espacio político. La ciudad como el lugar para las estrategias de participación. Polis, 7(20), 199-223. doi: 10.4067/S0718-65682008000100011

Baeza, M. (2003). Imaginarios sociales. Apuntes para la discusión teórica y metodológica. Concepción: Editorial Universidad de Concepción.

Blog Villa Claro de Luna. (2007, 21 de mayo). Diseño confeccionado por el arquitecto Matías Planas de la Municipalidad de Valdivia, que sería la futura plazuela de la Villa Claro de Luna. Disponible en http://villaclarodeluna.blogspot.com/2007_05_01_archive.html

Blog Villa Claro de Luna. (2007, 28 de mayo). Vecinos se reunieron en gran cantidad para hacer escuchar su voz. Disponible en http://villaclarodeluna.blogspot.com/2007_05_01_archive.html

$7 \mathrm{Al}$ modo como lo reseñara el historiador Guarda (1980). 
Blog Villa Claro de Luna. (2009, 12 de noviembre). Seminario reunió por primera vez las iniciativas ciudadanas de conservación de bosques y humedales urbanos de Valdivia. Disponible en http://villaclarodeluna.blogspot.com/2009_11_01_archive.html

Castoriadis, C. (1987). The imaginary institution of society. Cambridge, MA: The MIT Press.

Corral, M. (2010, 4 de febrero). La crisis de los humedales españoles. Diario El Mundo (España). Disponible en http://www.elmundo.es/elmundo/2010/02/01/ciencia/1265054602.html

Corvalán, E. \& Edy, M. (2003). Desarrollo local. Una metodología para la participación. Chile: Ediciones LOM/Fundación para la Superación de la Pobreza.

Cuenca, L. (2005). Celulosa Arauco en Valdivia: El desastre ambiental en el río Cruces, resultado del modelo forestal chileno. En M. Silva (Ed.), Entre el desierto verde y el pais productivo. El modelo forestal en Uruguay y el Cono Sur (pp. 35-43). Montevideo: Casa Bertolt Brecht y REDES-Amigos de la Tierra.

De San Eugenio, J. (2006). La interpretación del paisaje como instrumento de comunicación con la sociedad. Aportaciones de la semiótica y de los procesos de participación ciudadana. F@ro, 2(4), 1-13.

Diario Cultural. (2011, 26 de mayo). Se constituyó Comité Ecológico Angachilla. Disponible en http:// www.diariocultural.cl/index.php?option=com_content\&view $=$ article\&id=1494:se -constituyo-comite-ecologico-angachilla\&catid=57:valdivia\&Itemid $=106$

Díaz, F., Yáñez, L., Femenias, C., González, C., Huss, E., Mayorga ... \& Puentes, O. (2006). Plan Integral de Gestión Ambiental del Humedal de Rio Cruces. Valdivia: Corporación Nacional Forestal, Ministerio de Agricultura, Gobierno de Chile. Extraído el día 30 de junio de 2010 desde http://www.conaf.cl/cms/editorweb/rio_cruces/PIGA_resumen.pdf

Ferrero, A. \& Gargantini, D. (2004). El espacio local: Entre lineamientos políticos y concreciones. INVI, 19(50), 88-105. Disponible en http://www.revistapolitica.uchile.cl/index.php /INVI/article/viewArticle

Gervais-Lambony P., Landy F., \& Oldfield S. (2005). Reconfiguring identities and building territory in India and South Africa. New Delhi: Manohar.

Gilbert, L. \& Phillips, C. (2003). Practices of urban environmental citizenships: Rights to the city and rights to nature in Toronto. Citizenship Studies, 7(3),313-330. doi: 10.1080/1362102032000098896

Gissi, N. \& Soto, P. (2010). De la estigmatización al orgullo barrial: Apropiación del espacio e integración social de la población mixteca en una colonia de Ciudad de México. INVI, 25(68), 99-118. doi: 10.4067/S0718-83582010000100004

Gobernación de Valdivia. (2009, 29 de mayo). Reserva natural urbana de Villa Claro de Luna, luz verde para proyecto ecológico. Disponible en http://www.gobernacionvaldivia.gov.cl/n126_29 $-05-2009 . \mathrm{html}$

Guarda, G. (1980). Conjuntos urbanos histórico-arquitectónicos: Valdivia: ss. XVIII-XIX. Santiago: Nueva Universidad.

Instituto Nacional de Estadística (INE). (2005). Chile: Ciudades, pueblos, aldeas y caseríos. Santiago: Gobierno de Chile. Disponible en http://www.ine.cl/canales/chile_estadistico /demografia_y_vitales/demografia/demografia.php

Le Breton, D. (2007). El sabor del mundo. Una antropología de los sentidos. Buenos Aires: Nueva Visión.

Lotman, I., con Uspenskij, B. (2000). Sobre el mecanismo semiótico de la cultura. En I. Lotman, La semiosfera III. Semiótica de las artes y de la cultura (pp. 168-193). Madrid: Cátedra. 
Lungo, M. (2005). Globalización, grandes proyectos y privatización de la gestión urbana. Urbano, 8(11), 49-58. Disponible en http://redalyc.uaemex.mx/pdf/198/19801107.pdf

Ministerio de Medio Ambiente, Centro de Ecología Aplicada. (2011). Diseño del inventario nacional de humedales y el seguimiento ambiental. Ministerio de Medio Ambiente. Santiago: Ministerio de Medio Ambiente.

Ministerio de Vivienda y Urbanismo. (2010). Diseño de Parque Urbano. Valdivia: Secretaría Ministerial de Vivienda de la Región de los Ríos.

Molina, F., Montes, C., González, E., Rubio, J. \& Comisión de Seguimiento de la Gestión de los Humedales. (2002). El Plan Andaluz de Humedales. Una estrategia para la conservación de los humedales en el siglo XXI. Andalucía: Consejería de Medio Ambiente. Disponible en http://www.herbogeminis.com/IMG/pdf/plan_andaluz_humedales.pdf

National Research Council. (2001). Compensating for wetland losses under the Clean Water Act. Committee on Mitigating Wetland Losses, Board on Environmental Studies and Toxicology, Water Science and Technology Board, Division on Earth and Life Studies. Washington D.C.: National Academy Press.

Peck, J., Leitner, H., \& Sheppard, E. (2006). Contesting neoliberalism. The urban frontier. New York: The Guilford Press.

Ortiz, F., Piffaut, M., Pérez, C., Rojas, A., Cocq, F. \& González, A. (2009). Resultados preliminares de encuesta Comunidad Villa Claro de Luna. Valdivia: Fondecyt -F-1090465: "Los Paisajes del Agua”, Universidad Austral de Chile.

Osorio, F. (2009). Impacto del crecimiento urbano en el medio ambiente del humedal de Valdivia 19922007. Tesis para optar al grado de magíster en Asentamientos Humanos y Medio Ambiente. Instituto de Estudios Urbanos y Territoriales, Facultad de Arquitectura y Estudios Urbanos, Pontificia Universidad Católica de Chile.

Pulso Consultores S. A. (2006). Anteproyecto memoria nuevo plan regulador comuna de Valdivia. En Pulso Consultores S. A., Estudio Actualización Plan Regulador Comunal de Valdivia (pp. 1-75). Disponible en http://www.munivaldivia.cl/regulador/doc/anteproy_memoria.pdf

Pulso Consultores S. A. (s.f.). Humedales de la comuna de Valdivia. Consideraciones ambientales. Extraído el día 15 de julio de 2010 desde http://www.munivaldivia.cl/regulador/doc/taller _humedales.pdf

Ramsar (1994). Convención relativa a los humedales de importancia internacional, especialmente como hábitat de aves acuáticas. París, 13 de julio de 1994. Disponible en http://www.ramsar.org/cda/ es/ramsar-documents-texts-convention-on/main/ramsar/1-31-38\%5E20671_4000_2_

Rapoport, A. (1977). Aspectos humanos de la forma urbana. Hacia una confrontación de las ciencias sociales con el diseño de la forma urbana. Barcelona: Gustavo Gili.

Rojas, M., Campos, M., Alpízar, E., Bravo, J. \& Córdoba, R. (2003). El cambio climático y los humedales en Centroamérica: Implicaciones de la variación climática para los ecosistemas acuáticos y su manejo en la región. San José: Unión Mundial para la Naturaleza, Oficina Regional para Mesoamérica (UICN/ORMA).

Seager, J. (2000). Atlas del estado medioambiental. Madrid: Akal.

Tiner, R. (2005). In search of swampland. A wetland sourcebook and field guide. (2 $2^{\mathrm{a}} \mathrm{ed}$.). New Brunswick, N.J.: Rutdgers University Press. 
Toledo, A. (2005). Marco conceptual: Caracterización ambiental del Golfo de México. En A. Botello, J. Rendón, G. Gold-Bouchot \& C. Agraz-Hernández (Eds.), Golfo de México. Contaminación e impacto ambiental: Diagnóstico y tendencias. México: Universidad Autónoma de Campeche; Centro de Ecología, Pesquerías y Oceanografía del Golfo de México.

Toledo, A. (2006). Hacia una nueva visión de las relaciones entre el agua, el hombre y el paisaje. Gaceta Ecológica, 78, 5-10. Disponible en http://redalyc.uaemex.mx/pdf/539/53907801.pdf

Vargas, R. \& Piñeiro, N. (2005). El hidroscopio. México D. F.: Programa de las Naciones Unidas para el Medio Ambiente. Oficina Regional para América Latina y El Caribe, Red de Formación Ambiental.

Von Bertrab, A. \& Zambrano, L. (2010). Participatory monitoring and evaluation of a Mexico City wetland restoration effort. Ecological Restoration, 28(3), 343-353. doi:10.3368/er.28.3.343

Weingartner, G. (2001). Qualidade ambiental e gestão do espaço público: A política administrativa e o papel do sistema de espaços livres públicos no território urbano de Campo Grande-MS. Ensaios e Ciência, 5(3), 133-159. Disponible en http://redalyc.uaemex.mx/redalyc/src /inicio/ArtPdfRed.jsp?iCve=26050307\#

Zibechi, R. (2007). Autonomías y emancipaciones. América Latina en movimiento. Lima: Fondo Editorial de la Facultad de Ciencias Sociales, Universidad Nacional Mayor de San Marcos. 